\title{
Computing the Ruin Probability of Lévy Insurance Risk Processes in non-Cramér Models
}

\author{
Hyun Suk Park ${ }^{1, a}$ \\ ${ }^{a}$ Department of Finance and Information Statistics, Hallym University
}

\begin{abstract}
This study provides the explicit computation of the ruin probability of a Le $\phi$ vy process on finite time horizon in Theorem 1 with the help of a fluctuation identity. This paper also gives the numerical results of the ruin probability in Variance Gamma(VG) and Normal Inverse Gaussian(NIG) models as illustrations. Besides, the paths of VG and NIG processes are simulated using the same parameter values as in Madan et al. (1998).
\end{abstract}

Keywords: Lévy processes, failure time, Ruin probability, Variance Gamma, Normal Inverse Gaussian, Wiener-Hopf factorization.

\section{Introduction}

Let $X=\left\{X_{t}: t \geq 0\right\}, X_{0}=0$, be a Lévy process defined on $(\Omega, \mathcal{F}, P)$, with triplet $\left(\gamma, \sigma^{2}, \Pi_{X}\right), \Pi_{X}$ being the Lévy measure of $X$. For $x \in \mathbb{R}$, denote by $P_{x}(\cdot)$ the law of $X$ when it is started at $x$ and write simply $P_{0}=P$. The characteristic function of $X$ is given by the Lévy-Khintchine representation, $E\left(e^{i \theta X_{t}}\right)=e^{t \Psi(\theta)}$, where for $\theta \in \mathbb{R}$ and $t \geq 0$,

$$
\Psi(\theta)=\mathrm{i} \theta \gamma-\frac{\sigma^{2} \theta^{2}}{2}+\int_{\mathbb{R} \backslash\{0\}}\left(e^{\mathrm{i} \theta x}-1-\mathrm{i} \theta x \mathbf{1}_{\{|x|<1\}}\right) \Pi_{X}(\mathrm{~d} x) .
$$

In a number of numerical simulations and theoretical calculations for specific choices of Lévy processes, various authors have found that the stochastic models, viz, Insurance risk theory and American put optimal problem are solved as for the case that $X$ is a scaled Brownian motion with drifts and jumps. Various recent studies of insurance risk processes and associated random walks and Lévy processes have paid particular attention to the non-Cramér case, when upward jumps of the process may be very large. Such models are now thought to be quite realistic, especially in view of a recent tendency to large-claim events in the insurance industry. The occurrence of the claims in such a model is described by a point process and the amounts of money to be paid by the company at each claim by a sequence of random variables. The company receives a certain amount of premium to cover its liability. The company is assumed to have a certain initial capital $x$ at its disposal.

To give some intuition for the general framework of this paper, we briefly recall the classical insurance risk model. In the classical model, the claims arriving within the interval $(0, t], t>0$, are

This research was supported by Basic Science Research Program through the National Research Foundation of Korea(NRF) funded by the Ministry of Education, Science and Technology (2009-0071069). This research was supported by Hallym University Research Fund, 2010(HRF-2010-016).

${ }^{1}$ Associate Professor, Department of Finance and Information Statistics, Hallym University, Chuncheon 200-702, Republic of Korea. E-mail: hspark@hallym.ac.kr 
modeled as a compound Poisson process, yielding the risk process

$$
U_{t}=x+\gamma t-\sum_{i=1}^{N_{t}} Y_{i}, \quad t \geq 0,
$$

where $x$ is the initial risk capital and $\gamma>0$ is the premium rate. Denote by $F$ the claim size distribution function, that is, the distribution function of the independent and identically distributed almost surely positive random variables $Y_{i}$, assumed to have mean $\mu>0$. Let $\lambda>0$ be the intensity of the Poisson process.

Under this scenario, one important problem in insurance risk theory is to investigate the "ruin probability" i.e., the probability that the risk business ever becomes negative. The probability of ruin is then

$$
\begin{aligned}
P\left(U_{t}<0 \text { for some } t>0\right) & =P\left(\sum_{i=1}^{N_{t}} Y_{i}-\gamma t>x, \text { for some } t>0\right) \\
& =(1-\rho) \sum_{n=1}^{\infty} \rho^{n} \overline{F_{I}^{* n}}(x),
\end{aligned}
$$

where $\rho=\lambda \mu / \gamma<1$, and Equation (1.3) follows from ladder height analysis. The integrated tail distribution $F_{I}(u):=1 / \mu \int_{(0, u]} \bar{F}(y) \mathrm{d} y, u \geq 0$, is the distribution function associated with the increasing ladder height process of the process $X_{t}=\sum_{i=1}^{N_{t}} Y_{i}-\gamma t, t \geq 0$, and $\overline{F_{I}^{* n}}$ is the tail of its $n$-fold convolution.

This corresponds to an insurance risk model with premiums and other income producing a downward drift in $X$, while claims are represented by positive jumps: see Grandell (1991). Thus "ruin" occurs at a positive level $x$, if this is reached by $X$. In the finite time horizon we will show the ruin probability of the process above a level $x$. We assume Lévy process $X$ drifts to $-\infty$ almost surely. In this paper our aim is to estimate the limiting ruin probability of an infinite time horizon of quantities of interest in insurance risk analysis, and to consider the explicit ruin probability of finite time horizon in Theorem 1. Specific illustrations of the theoretical results are given for special cases such as VG and NIG models. In this analysis we will follow the notation of Park and Maller (2008).

\section{Preliminaries}

The fluctuation theory provides a number of formulas for the distribution of certain random variables related to extrema of a Lévy process. An important tool in the study of the fluctuations of Lévy processes is the following Wiener-Hopf factorization which we review for convenience. For a more detailed account, the reader is referred to Section 2 of VI in Bertoin (1996). Assume that $q>0$. Let $\tau_{q}$ be an exponentially distributed random time with parameter $q$, which is independent of Lévy process. We have that $\bar{X}_{\tau_{q}}$ and $\bar{X}_{\tau_{q}}-X_{\tau_{q}}$ are independent where $\bar{X}_{t}=\sup _{0 \leq s \leq t} X_{s}$ and $\tau_{q}$ is an independent exponentially distributed random variable with parameter $q$. As a consequence, for $\theta \in \mathbb{R}$,

$$
\frac{q}{q+\Psi(\theta)}=\Psi_{q}^{+}(i \theta) \cdot \Psi_{q}^{-}(i \theta)
$$

with

$$
\Psi_{q}^{+}(i \theta)=E\left[e^{i \theta \bar{X}_{\tau_{q}}}\right] \quad \text { and } \quad \Psi_{q}^{-}(i \theta)=E\left[e^{-i \theta\left(\bar{X}_{\tau_{q}}-X_{\tau_{q}}\right)}\right]=E\left[e^{i \theta \underline{X}_{\tau_{q}}}\right]
$$


where $\underline{X}_{t}=\inf _{0 \leq s \leq t} X_{s}$.

We now introduce some basic notations, definitions and results, which consist of some renewaltheoretic aspects of Lévy processes. Our results can be seen as adding to an understanding of renewal and fluctuation properties of Lévy processes which drift to $-\infty$. Define the ascending ladder height subordinator of $X$ by $\left(H_{t}\right)_{t \geq 0}$, which is defective under the assumption $\lim _{t \rightarrow \infty} X_{t}=-\infty$ a.s. (which we will always assume in this paper), taking the value $+\infty$ once $X$ exceeds its all-time maximum. The (proper) descending ladder height subordinator is denoted by $\left(\widehat{H}_{t}\right)_{t \geq 0}$ taking positive values as Doney and Kyprianou (2006). Denote by $\left\{\left(L_{t}^{-1}, H_{t}\right): t \geq 0\right\}$ and $\left\{\left(\widehat{L}_{t}^{-1}, \widehat{H}_{t}\right): t \geq 0\right\}$ the bivariate subordinators representing the increasing and decreasing ladder processes. Associated with the increasing and decreasing ladder processes are the bivariate renewal measures defined by

$$
\mathcal{V}(\mathrm{d} x, \mathrm{~d} s)=\int_{(0, \infty)} \mathrm{d} t \cdot P\left(H_{t} \in \mathrm{d} x, L_{t}^{-1} \in \mathrm{d} s\right),
$$

and taking Laplace transforms shows that

$$
\int_{(0, \infty)} \int_{(0, \infty)} e^{-p x-r s} \mathcal{V}(\mathrm{d} x, \mathrm{~d} s)=\frac{1}{\kappa(p, r)}, \quad \text { for } p, r \geq 0,
$$

where we denote by $\kappa(p, r)$ their joint Laplace exponents such that

$$
\kappa(0, r)=q+c r+\int_{(0, \infty)}\left(1-e^{-r x}\right) \Pi_{\mathcal{H}}(\mathrm{d} x),
$$

where $\mathcal{H}$ is a nondefective process and $H$ is obtained from $\mathcal{H}$ by exponential killing with rate $q>0$ so that $q>0$ if and only if $\lim _{t \rightarrow \infty} X_{t}=-\infty, c \geq 0$ is the drift of $\mathcal{H}$ and $\Pi_{\mathcal{H}}$ is its jump measure. We denote the following marginal measure of $\mathcal{V}(\cdot, \cdot)$ by the (defective, when $\lim _{t \rightarrow \infty} X_{t}=-\infty$ a.s.) process $\mathcal{H}$ :

$$
V(\mathrm{~d} x):=\mathcal{V}(\mathrm{d} x,[0, \infty))=\int_{(0, \infty)} P\left(\mathcal{H}_{t} \in \mathrm{d} x\right) e^{-q t} \mathrm{~d} t, \quad \text { for } x \geq 0 .
$$

Similar notation will also be used for $\widehat{\mathcal{V}}(\cdot, \cdot)$. We denote the Lévy measure of $\mathcal{H}$ by $\Pi_{\mathcal{H}}$, and similarly for $\widehat{H}$. The tails of $\Pi_{X}$ are

$$
\bar{\Pi}_{X}^{+}(x)=\Pi_{X}\{(x, \infty)\}, \quad \bar{\Pi}_{X}^{-}(x)=\Pi_{X}\{(-\infty,-x)\}
$$

and

$$
\bar{\Pi}_{X}(x)=\bar{\Pi}_{X}^{+}(x)+\bar{\Pi}_{X}^{-}(x), \quad x>0,
$$

with similar notations $\bar{\Pi}_{\mathcal{H}}(x)$ and $\bar{\Pi}_{\widehat{H}}(x)$ for $\mathcal{H}$ and $\widehat{H}$.

For our non-Cramér scenario, it will be necessary to introduce some more notation. For $\alpha \geq 0$, we shall say that a distribution $F$ on $[0, \infty)$ with tail $\bar{F}$ belongs to the class $\mathcal{L}^{(\alpha)}$, if

$$
\lim _{u \rightarrow \infty} \frac{\bar{F}(u-x)}{\bar{F}(u)}=e^{\alpha x}, \quad \text { for } x \in(-\infty, \infty) .
$$

Tail functions $\bar{F}$ such that $\bar{F}(\log u)$ is regularly varying with index $-\alpha, \alpha \geq 0$, as $u \rightarrow \infty$, are in $\mathcal{L}^{(\alpha)}$ : see Bingham et al. (1987), Embrechts et al. (1979) and Klüppelberg (1989). With $*$ denoting 
convolution, a distribution $F$ is said to be convolution equivalent, i.e., in the class $\mathcal{S}^{(\alpha)}, \alpha \geq 0$, if $F \in \mathcal{L}^{(\alpha)}$, and, in addition,

$$
\lim _{x \rightarrow \infty} \frac{\overline{F^{2 *}}(x)}{\bar{F}(x)}:=2 \delta_{\alpha}(F)<\infty,
$$

where $\delta_{\alpha}(F):=\int_{(0, \infty)} e^{\alpha x} F(\mathrm{~d} x)$. The parameter $\alpha$ is referred to as the index of the class. When $\alpha=0$, $\mathcal{S}:=\mathcal{S}^{(0)}$ is the class of subexponential distributions.

At this stage, we bring in the convolution equivalent assumptions. We assume the same conditions as in Park and Maller (2008), namely, that

$$
\lim _{t \rightarrow \infty} X_{t}=-\infty \text { a.s. and } e^{-q} \delta_{\alpha}(\mathcal{H})<1 .
$$

Throughout the entire paper the relationship of the above assumption is mentioned well in Section 4 of Klüppelberg et al. (2004). Assume that the process is not spectrally negative, in fact, assume $\Pi_{X}\{(x, \infty)\}>0$ for all $x>0$. The second assumption means that the measure is in $\mathcal{L}^{(\alpha)}$ or $\mathcal{S}^{(\alpha)}$ if this is true for the corresponding distribution tail. In fact, our main assumption throughout this section will be $\bar{\Pi}_{\mathcal{H}} \in \mathcal{S}^{(\alpha)}$, for a specified $\alpha>0$.

\section{Ruin Probability}

We consider in this section the estimation of limiting ruin probability of an infinite time horizon and the explicit ruin probability of a finite time horizon. Park and Maller (2008) and Klüppelberg et al. (2004) introduced the limiting probability when causes the ruin as follows:

$$
\lim _{u \rightarrow \infty} P\left(\tau_{u}<\infty\right)=\frac{q}{\left(q-\log \delta_{\alpha}(\mathcal{H})\right)^{2}} \bar{\Pi}_{\mathcal{H}}(u)
$$

To a fixed level $x \in \mathbb{R}$ we associate the first strict passage time $\tau_{x}$ (resp. $\tau_{x^{-}}$) above (resp. below) $x$, that is,

$$
\tau_{x}=\inf \left\{t \geq 0: X_{t}>x\right\} \text { and } \tau_{x^{-}}=\inf \left\{t \geq 0: X_{t}<-x\right\} .
$$

Theorem 1. Let $\tau_{q}$ be an exponentially distributed random time with parameter $q>0$, which is independent of the Lévy process. We then obtain for $\alpha>0$ and $x \geq 0$,

$$
E\left(e^{-\alpha \tau_{x}} 1_{\left[\tau_{x}<\infty\right]}\right)=E\left(1_{\left[\bar{X}_{\tau_{q}}>x\right]}\right)
$$

and

$$
P\left(\tau_{x}>t\right)=\frac{1}{q} \kappa(q, 0) \int_{(0, x]} P\left(\mathcal{H}_{t} \in \mathrm{d} y\right) .
$$

Proof: Substituting $\beta=0$ to Lemma 1 of Alili and Kyprianou (2005), we have easily the result (3.1). Assume that $\alpha, x>0$ and note that $E\left[1_{\left(\bar{X}_{\tau_{q}}>x\right)}\right]=E\left[1_{\left(\tau_{x}<\tau_{q}\right)}\right]$. By taking Laplace transforms of both sides of (3.1) and using Fubini's theorem, we can write, for $\lambda>0$,

$$
\begin{aligned}
\int_{(0, \infty)} e^{-\lambda x} E\left(e^{-\alpha \tau_{x}} 1_{\left[\tau_{x}<\infty\right]}\right) \mathrm{d} x & =\int_{(0, \infty)} e^{-\lambda x} E\left(1_{\left[\bar{X}_{\tau_{q}}>x\right]}\right) d u \\
& =\frac{1}{\lambda} \int_{(0, \infty)}\left(1-e^{-\lambda y}\right) P\left(\bar{X}_{\tau_{q}} \in \mathrm{d} y\right) .
\end{aligned}
$$


Therefore we have

$$
\lambda \int_{(0, \infty)} e^{-\lambda x} P\left(\tau_{x}<\tau_{q}\right) \mathrm{d} x=1-E\left(e^{-\lambda \bar{X}_{\tau_{q}}}\right)
$$

From Bertoin (1996), p.172, we have

$$
\begin{aligned}
\lambda \int_{(0, \infty)} e^{-\lambda u} P\left(\tau_{x}>\tau_{q}\right) d u & =\lambda \int_{(0, \infty)} e^{-\lambda x}\left(\int_{(0, \infty)} P\left(\tau_{x}>t\right) q e^{-q t} \mathrm{~d} t\right) \mathrm{d} x \\
& =\frac{\kappa(q, 0)}{\kappa(q, \lambda)} .
\end{aligned}
$$

Hence for every $\lambda>0$, we obtain from (2.2) and (3.3)

$$
\int_{(0, \infty)} e^{-\lambda x}\left(\int_{(0, \infty)} P\left(\tau_{x}>t\right) q e^{-q t} \mathrm{~d} t\right) \mathrm{d} x=\int_{(0, \infty)} e^{-\lambda x}(\kappa(q, 0) V(x)) \mathrm{d} x .
$$

By the uniqueness of Laplace transform, we then get (3.2) as follows:

$$
\int_{(0, \infty)} P\left(\tau_{x}>t\right) q e^{-q t} \mathrm{~d} t=\kappa(q, 0) V(x) .
$$

By replacing $X$ by $-X$ in the previous theorem, we get the following result.

Corollary 1. For all $\alpha>0$ and $x \geq 0$, we have

$$
E\left(e^{-\alpha \tau_{x^{-}}} 1_{\left[\tau_{x^{-}}<\infty\right]}\right)=E\left(1_{\left[\underline{X}_{\tau q}<-x\right]}\right) .
$$

Next we consider spectrally positive processes. We assume that $X$ drifts to $-\infty$ a.s., and the downward ladder height process is simply a negative linear drift, $\widehat{H}_{t}=-t, \bar{\Pi}_{\mathcal{H}}(u)=\int_{y \in[u, \infty)} \bar{\Pi}_{X}^{+}(y) \mathrm{d} y<$ $\infty$, for $u>0, E\left|X_{1}\right|<\infty$ and $E X_{1}<0$. Suppose that $X$ has Laplace exponent $\phi(\theta)$ for $\theta \in \mathbb{R}$ as follows: $E\left(e^{\theta X_{t}}\right)=e^{\phi(\theta) t}$. We assume that $\bar{\Pi}_{X}^{+} \in \mathcal{S}^{(\alpha)}$, for $\alpha>0$. We know that $\phi$ and $\Psi$ are related through the identity $\phi(\theta)=-\Psi(-i \theta)$ : see Bertoin (1996), Chapter VII and Sato (1999). Taking the fact that the downward ladder height process is a linear drift, we can identify $q$ as follows: $q=\lim _{\theta \rightarrow 0} \phi(-\theta) / \theta$. Note that $q=-\phi^{\prime}(0-)=\left|E X_{1}\right|<\infty$, and $q-\log \delta_{\alpha}(\mathcal{H})=-\phi(\alpha) / \alpha$, for $\alpha>0$. It is known that $\widehat{q}=0$ and $\widehat{V}(\mathrm{~d} x)=-\mathrm{d} x$, when $X$ has bounded variation and drifts to minus infinity from Klüppelberg et al. (2004).

Corollary 2. Suppose that $X$ is spectrally positive, drifts to $-\infty$ a.s., satisfies the assumption (2.7) for a given $\alpha>0$, and has $\phi(\alpha)<0$. Then we have:

$$
\lim _{x \rightarrow \infty} P\left(\tau_{x}<\infty\right)=\left|E X_{1}\right|\left(\frac{\alpha}{\phi(\alpha)}\right)^{2} \int_{y \in[u, \infty)} \bar{\Pi}_{X}^{+}(y) \mathrm{d} y .
$$

For the special case of jump diffusion process, we have

$$
\phi(\alpha)=-\alpha \gamma+\frac{\sigma^{2} \alpha^{2}}{2}+\int_{y \in[0, \infty)}\left(e^{\theta y}-1\right) \Pi_{X}(\mathrm{~d} y),
$$

which is finite by our assumption. Take $\alpha>0$. We can calculate easily that assuming $\rho=\mu \lambda / \gamma<1$,

$$
\left|E X_{1}\right|=\gamma(1-\rho), \quad \frac{-\phi(\alpha)}{\alpha}=\gamma\left(1-\frac{\sigma^{2} \alpha}{2 \gamma}-\frac{1}{\alpha \gamma} \int_{y \in(0, \infty)}\left(e^{\alpha y}-1\right) \Pi_{X}(\mathrm{~d} y)\right) .
$$




\section{Numerical Study}

We now give some numerical illustrations of the limiting behavior of distributions of quantities interested in Lévy insurance risk processes. We conclude this section with some examples, all of which have the shape that $X$ is spectrally positive; namely, we assume $\bar{\Pi}_{X}^{-}=0$. This case is very tractable and allows us to derive quite explicit expressions which generalize well-known results in collective risk theory. It is the case of most direct interest in insurance applications.

The numerical study is illustrated for the Variance Gamma(VG) and Normal inverse Gaussian(NIG) models. For that purpose the parameters of our VG and NIG models are obtained using Fourier transforms in Madan et al. (1998), where the data for their study were obtained from the Financial Futures Institute in Washinton D.C. and include all time stamped transaction option prices from January 1992 to September 1994. For parameter estimation, the data employed was the 691 daily observations of $\log$ spot price relatives covering the period from January 1992 to September 1994. Using all of the option prices available for each week, they also estimated the parameter values for VG models on a weekly basis. The details are given by Section 5 of Madan et al. (1998).

Suppose that $X_{t}$ is of the form

$$
\mathrm{VG}_{t}=\sigma W_{g(t ; v)}+\gamma g(t ; v), \quad t \geq 0,
$$

where $W_{t}$ is a standard Brownian motion, and $g(t ; v)$ is a Gamma process. Madan et al. (1998) introduce this process defined by a Brownian motion with drift $\gamma$ and volatility $\sigma$, time-changed by an increasing Gamma process with unit mean and variance $v$. From three representations of the Lévy measure for VG process in Madan et al. (1998), we may choose a measure changed by a constant relative risk utility function as in Madan and Milne (1991). Throughout the Variance Gamma model, Lévy density can be expressed as

$$
\Pi_{X}(\mathrm{~d} x)=\frac{1}{v|x|} \exp \left(-\frac{|x|}{\sigma} \sqrt{\frac{2}{v}+\frac{\gamma^{2}}{\sigma^{2}}}\right) \cdot \exp \left(\frac{\gamma x}{\sigma^{2}}\right) \mathrm{d} x, \quad-\infty<x<\infty .
$$

The parameters we use for the VG model are the same as used in Madan et al. (1998). In Maller et al. (2006) the parameters for NIG model were obtained by matching its first four moments to those of the VG model. Under the VG model, the parameters we use in (4.2) are estimated as in Maller et al. (2006): $v=0.20, \gamma=-0.14$ and $\sigma=0.12$. These parameters we chosen so that $\Psi(1)=0.10$ for our purpose. Simulated results of the path of VG process, a pure jump process of finite variation, with infinitely many jumps are given by Figure 1. This process is constructed by means of "Brownian subordinator" by evaluating the Brownian motion with drift at random times given by a Gamma process. There are three basic controls: the volatility and drift of the Brownian motion and the variance of the Gamma process. These parameters allow the control of the skewness and kurtosis of the claim distribution in addition to mean and variance. Figure 1 presents the path of simulating VG as the Gamma time-changed Brownian motion for sequentially generating VG sample paths on $[0, T]$ at time points $0=t_{0}<t_{1}<\cdots<t_{N-1}<t_{N}=T$, where the time spacings $\Delta t_{i}$, $i=1, \ldots, N$ are given as inputs, along with VG parameters. For more information on efficiently generating samples from VG, see Fishman (1996).

Define $T_{(t ; v)}$ the first time that a Brownian motion with drift $v$ reaches the positive level $t$. We consider the Brownian motion with drift $\gamma$ and volatility $\sigma$ computed at this Gaussian time. Then we can define the NIG process by

$$
\mathrm{NIG}_{t}=\sigma W_{T_{(t ; v)}}+\gamma T_{(t ; v)}, \quad t \geq 0
$$




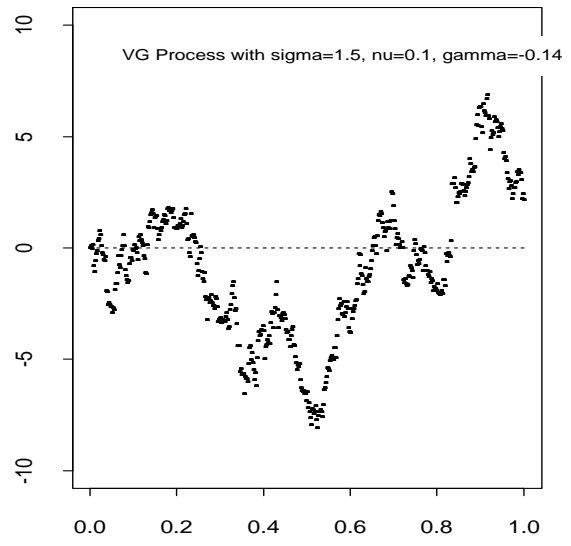

(a)

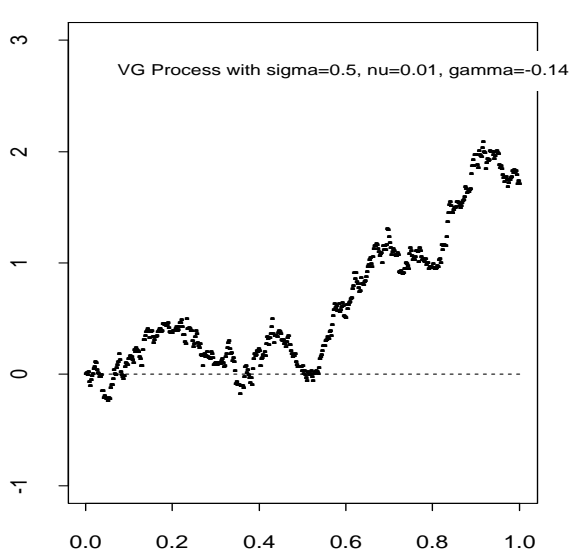

(b)

Figure 1: Infinite activity Lévy processes: Variance Gamma $V G_{t}=\sigma W_{g(t ; v)}+\gamma g(t ; v)$, where $W_{t}$ is $S B M$ and $g(t ; v)$ is a Gamma process

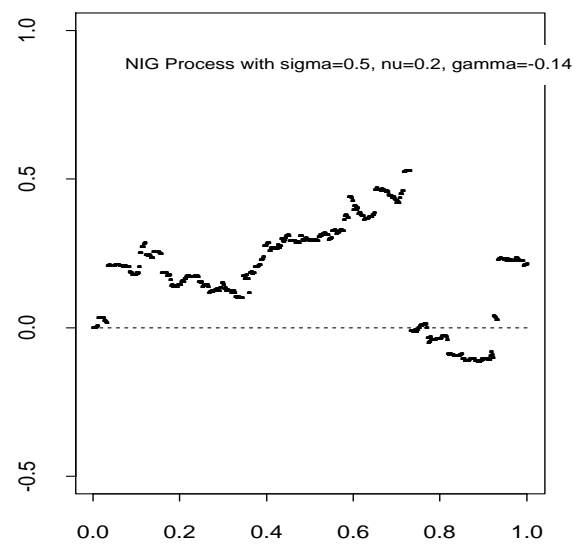

(a)

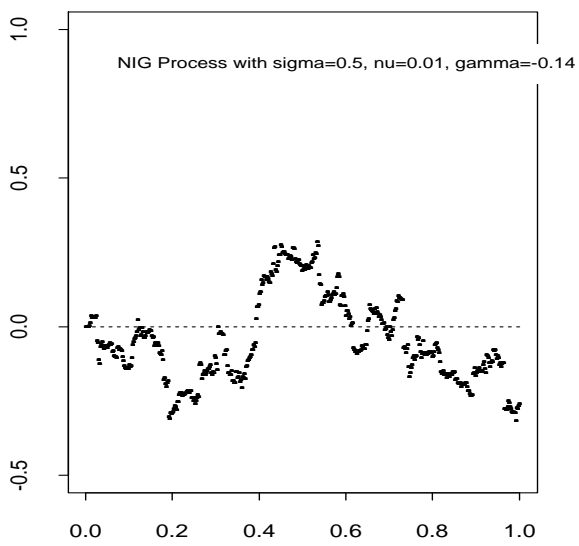

(b)

Figure 2: Infinite activity Lévy processes: Normal Inverse Gaussian $N I G_{t}=\sigma W_{T_{(t ; v)}}+\gamma T_{(t ; v)}$

The Lévy measure for the NIG model can be expressed as

$$
\Pi_{N I G}(\mathrm{~d} x)=\frac{\delta \omega}{\pi} \exp (\beta x) \frac{K_{1}(\omega|x|)}{|x|},
$$

where $\beta=\gamma / \sigma^{2}, \omega=\sqrt{v^{2} / \sigma^{2}+\gamma^{2} / \sigma^{4}}, \delta=\sigma$ and $K_{1}$ is the modified Bessel function with index 1 such that $K_{1}(x)=x / 2 \int_{t \in(0, \infty)} \exp \left(-\left(t+x^{2} / 4 t\right)\right) t^{-2} \mathrm{~d} t$. In Maller et al. (2006), they set $\omega=28.421$, $\beta=-15.086$ and $\delta=0.317$. In Figure 2, the NIG process is in many ways similar to the VG process due to Madan and Seneta (1990). This simulation result shows a path of the NIG process at various moments in time. The NIG process is a pure-jump process with infinite variation. In the case of the VG process the time change process is the Gamma process; in the case of a NIG process it is an inverse Gamma process. Both processes are pure-jump Lévy processes (they have no continuous Brownian component), but they differ in the nature of jumps: the VG process has jumps of finite variation while 
Table 1: $\lim _{x \rightarrow \infty} P\left(\tau_{x}<\infty\right)$ for $\alpha=0.1,1.0,2.0$.

\begin{tabular}{cccccccc}
\hline \hline Initial capitals & \multicolumn{3}{c}{ VG Model } & & \multicolumn{3}{c}{ NIG Model } \\
\cline { 2 - 4 } \cline { 6 - 8 } & $\alpha=0.1$ & $\alpha=1.0$ & $\alpha=2.0$ & & $\alpha=0.1$ & $\alpha=1.0$ & $\alpha=2.0$ \\
\hline 5 & 0.994886 & 0.975121 & 0.948513 & & 0.999985 & 0.990167 & 0.968494 \\
10 & 0.779851 & 0.667903 & 0.649678 & & 0.999736 & 0.980240 & 0.958785 \\
50 & 0.105767 & 0.070577 & 0.068651 & & 0.920581 & 0.902629 & 0.882873 \\
100 & 0.010806 & 0.006737 & 0.006553 & & 0.826228 & 0.810116 & 0.792384 \\
300 & 0.000002 & 0.000001 & 0.000001 & & 0.499817 & 0.490070 & 0.479344 \\
900 & 0.000000 & 0.000000 & 0.000000 & & 0.010200 & 0.010000 & 0.009783 \\
1000 & 0.000000 & 0.000000 & 0.000000 & & 0.000000 & 0.000000 & 0.000000 \\
\hline \hline
\end{tabular}

the variation of the NIG process is infinite. The parameters are the drift and volatility of the timechanged Brownian process and the variance of the time-change process. Program language $C^{++}$and other commercial software S-plus are generally used for visualizations.

Now we consider the limiting distribution of ruin on infinite time horizon as numerical illustrations. Using the simulating results of VG and NIG processes in Figures 1 and 2, we can estimate the expected value of $X_{1}$. Then we will set up that the intensity of the Poisson process in classical risk process (1.2) is $\lambda=0.3$, and the mean of claim size is $\mu=0.3$. Also, we adjust a range of the initial capital level $x$ to a range $(0,1000)$ which means that 1000 is an infinity level. Let us proceed to examine the limiting behavior the ruin probability of (3.6) as $x \rightarrow \infty$ for VG and NIG models in more detail in Table 1. According to the index $\alpha$ and two kinds of models, the limiting behavior of the ruin probability looks like a different shape. The steep decreasing slope as $x \rightarrow \infty$ means that the much more large claims occur and the distribution of claim has the more heavy-tailedness distribution class.

\section{References}

Alili, L. and Kyprianou, A. E. (2005). Some remarks on first passage of Lévy processes, the Americal put and pasting principles, The Annals of Applied Probability, 15, 2062-2079.

Bertoin, J. (1996). Lévy Processes, Cambridge University Press.

Bingham, N. H., Goldie, C. M. and Teugels, J. L. (1987). Regular Variation, Cambridge University Press, Cambridge.

Doney, R. A. and Kyprianou, A. (2006). Overshoots and undershoots of Lévy processes, The Annals of Applied Probability, 16, 91-106.

Embrechts, P., Goldie, C. M. and Veraverbeke, N. (1979). Subexponentiality and infinite divisibility, Probability Theory and Related Fields, 49, 335-347.

Fishman, G. S. (1996). Monte Carlo Methods: Concepts, Algorithms, and Applications, Springer.

Grandell, J. (1991). Aspects of Risk Theory, Springer-Verlag, New York.

Klüppelberg, C. (1989). Subexponential distributions and characterizations of related classes, Probability Theory and Related Fields, 82, 259-269.

Klüppelberg, C., Kyprianou, A. and Maller, R. (2004). Ruin probability and overshoots for general Lévy insurance risk processes, The Annals of Applied Probability, 14, 1766-1801.

Madan, D., Carr, P. and Chang, E. (1998). The variance gamma process and option pricing model, European Finance Review, 2, 79-105.

Madan, D. and Milne, F. (1991). Option pricing with VG martingale components, Mathematical Finance, 1, 39-55.

Madan, D. and Seneta, E. (1990). The variance gamma process model for share market returns, Journal of Business, 63, 511-524. 
Maller, R., Solomon, D. and Szimayer, A. (2006). A multinomial approximation for American option prices in Lévy process models, Mathematical Finance, 160, 613-633.

Park, H. S. and Maller, R. (2008). Moment and MGF convergence of overshoots and undershoots for Lévy insurance risk processes, Advances in Applied Probability, 40, 716-733.

Sato, K. (1999). Lévy Processes and Infinitely Divisible Distributions, Cambridge University Press, Cambridge. 